\title{
Anatomical Coordinate System for Bilateral Registration of Mammograms
}

\author{
F redrik Georgsson \\ Umeå University, Department of Computing Science, SE-901 87 UMEA, Sw eden, \\ fredrikg@cs.umu.se, \\ http://www.cs.umu.se/ fredrikg/ic_iman.htm
}

\begin{abstract}
One important issue when judging mammograms are bilateral comparison. In this pap er, someasp ects on the problem of determining coordinates of points inrelation to the anatomy of the breast is given. The $\mathrm{p}$ oin ts are expressedin anatomicalcoordinates, making it p ossibleto compare mammograms without doing an yeometrical transformations of the images. The method is implemented and is fully automatic.
\end{abstract}

\section{Introduction and Previous Work}

F or radiologists judging mammograms in general and screening mammograms in particular it is very important to haveaccess to sev eralimages from the same $\mathrm{w}$ oman.These images are b oth pro jectionsof the same breast tak enat different occasions (temporal) and images of the tw obreasts (bilateral). In bo th cases un usualchanges b etw eerthe images cause suspicion. In this paper, a framework for bilateral comparison of mammograms in an anatomical co ordinatesystem is deriv edand described.

The issue of bilateral comparison has been studied for a long time. Among the previous w orkthat has been carried out it is p ossible to iden tifythree major approaches; one in which w e d not transform the images but compare top ological properties of different regions [9, 10,14], one in which w e atually transform the images, enabling p oin t-wisecomparison $[5,12,16]$ and finally, some methods which transform the breast edge or p erform affine transformations of the images $[11,13]$.

The method presented in this w orkis classified b etw eerthe first and second approach since it compares areas of the images but still allo ws for po int-wise comparison.

\section{Outline of the Method}

Normally w eassign co ordinates to po ints in an image. These co ordinates are giv en in relation to some orthonormal base vectors, typically aligned with the rows and columns of the image, see $\mathcal{C}$ in Figure 1, left. The disadvan tageswith expressing co ordinates in relation to the image frame and not ob jects within the image are ob vious. More stable co ordinates are obtained if the co ordinate 

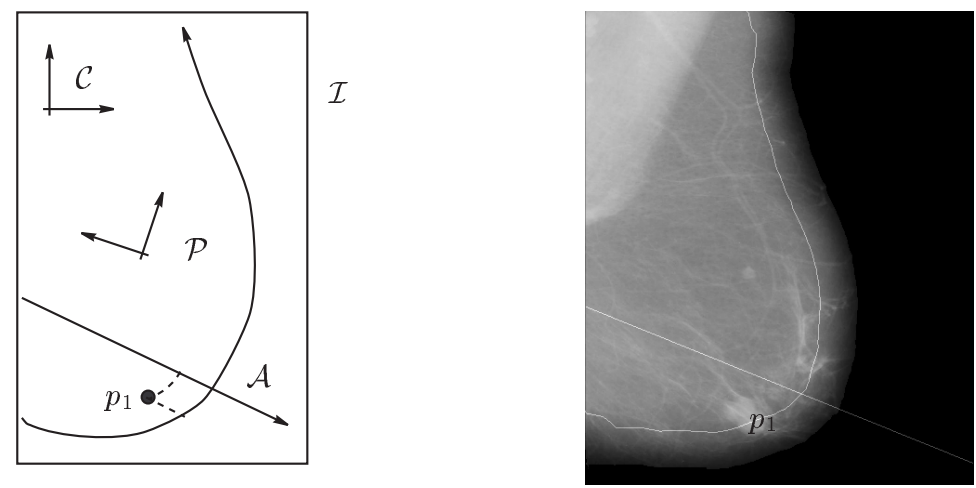

Fig. 1. Left: Different coordinate systems. $\mathcal{C}$ is the normal system that relates coordinates to the image frame $\mathcal{I}$ and not the object within the image. Its axes are orthonormal vectors. The coordinate system $\mathcal{P}$ is still an orthonormal system, but oriented in relation to the object depicted and not the image frame. The coordinate system $\mathcal{A}$ is expressed in relation to anatomical features and its axis are not orthogonal they can even be curved. Right: A point $p_{1}$ and the corresponding equidistance line (the curved line). The straight line is the nipple line. Mammogram 5 from the MIAS database.

axes are aligned with objects in the image. This is rather straightforward to do with, for instance, principal component techniques. The resulting axis are still orthonormal vectors, see $\mathcal{P}$ in Figure 1, left . The idea with the method proposed here is to express the coordinates of a point $p$ in relation to anatomical features hence making the coordinates even more stable. The reference features define an anatomical coordinate system although with non-linear coordinate axes, see $\mathcal{A}$ in Figure 1, left. If this coordinate system is chosen carefully the hypothesis is that, it should be possible to compare the coordinates of points from bilateral (left-right) breasts.

The anatomical coordinates are given in relation to the distance to the skinline and the distance to the nipple-line along an equidistance line, see Figure 1, right. The nipple line is the line perpendicular to a straight-line approximation of the pectoralis that passes through the nipple. The skin line is the projection of the breast-air interface on to the image plane.

An obvious advantage of the anatomical based coordinate system is that the positioning of the breast at the time of image acquisition is not critical. Another advantage is that the coordinate system moves with the breast, decreasing the effect of non-sufficient stretching of the breast prior to compression. There are some indications [6], that the coordinate system aligns with the tissue movement while under compression, but this still remains to be completely verified. It has, however, been shown that the position of the nipple point on the skin-line to a high degree corresponds to the point with maximum distance to the pectoralis line [15]. This indicates an anatomical suitability of the coordinate system. 
The idea of how to use the anatomical coordinates is quite simple: for sets of points, for instance $p_{1}$ in one image, calculate their anatomical coordinates and compare properties of the local surroundings, i.e. $\mathcal{N}_{p_{1}}$, with properties of the corresponding points surrounding $\mathcal{N}_{p_{2}}$. The points that have a low degree of similarity are considered suspicious.

Given the anatomical coordinates it is possible to compare a large set of parenchyma properties: texture, distribution of masses and even the spatial distribution of the glandular disc. The parenchyma is a composition of tissue specific for a certain organ, for instance the breast. The glandular disc is the projection of the glandular tissue.

\section{Distances in digital images}

A key issue in the proposed method is the ability to calculate the distance between a point and two different lines in the image: the skin line and the nipple line.

The equidistance curve is defined to be all points $\left\{p_{i}\right\}_{1}^{N}$ that have distance $\delta$ to the border of the object. The most efficient way to find the equidistance lines is to use the Distance Transform, described in detail in [1]. This is the method used in this paper with weights $a=0.955$ and $b=1.37$.

Once the equidistance line that passes through an interesting point $p$ has been established it is required to calculate the distance along this line to the intersection of the nipple line. It is possible to identify three different problems in doing this. The first problem is that it might be two or more intersections between the equidistance line and the nipple line. The reason for this is that if the breast has not been properly stretched at image acquisition there might be a concavity at the infra mammary sulcus. This problem is solved by defining the intersection between the two lines that is closest to the nipple as the 'correct' intersection. This intersection defines a point $r$ on the equidistance line and the distance along the equidistance line and $p$ gives the desired coordinate.

The second problem is that if the two lines are supposed to be eight connected they can intersect each other without having pixels in common. Suppose that the lines $L=\left\{p_{i}\right\}_{1}^{N}$ and $L_{2}=\left\{q_{i}\right\}_{1}^{M}$ are suspected to intersect each other but $L_{1} \cap L_{2}=\emptyset$ we can find candidates for intersection points in the set $I=L_{1} \cap$ $\left(L_{2} \oplus B\right)$ where $B$ is a structuring element of a eight-connected neighbourhood and $\oplus$ is the morphological dilate-operation.

The point $r$ divides the equidistance line into two parts, the upper part $U$ and the lower part $L$ of the breast. The part that contains the point $p$ is kept and a line trace starts at $r$.

The distance along the equidistance line is computed by counting the number of pixels separating the two points along the line and the weighting the verticalhorizontal distance with $a$ and the diagonal distances with $b$. It can be shown that optimal values, in the case of uncorrelated directions of adjacent pixels, are $a=0.9445$ and $b=1.3459$, respectively [7]. 


\section{Detection of anatomical features}

\subsection{Detection of the skin line}

The equidistance lines are determined in relation to the skin line and hence it is very important to determine this line with relatively high accuracy.

In the current version of this work, a simple threshold method is used. The mammogram is thresholded at a low intensity level with a global threshold. The binary image is then searched for connected components. The breast is typically found as one object and the meta-information, such as the name of the patient, projection, and side, are found as separate connected components. By using the fact that the position of the breast is known and that the breast is the largest connected component in the image it can be determined which of the components that is the breast.

The extracted binary breast object is then post processed by performing a morphological close operation. It is important to use a closing and not an opening, since a closing smoothes the border by adding pixel while an opening smoothes the border by removing pixels. If pixels are allowed to be removed from the segmented breast object, some internal points of the breast could be removed and this is not desirable.

A pixel is said to belong to the skin line if it belongs to the background has at least one neighbour that belongs to the breast.

\subsection{Detection of the pectoralis}

In the literature several ways of finding the pectoralis are described. In this paper a method developed by Karssemeijer is used [8]. This method finds a straight-line approximation of the pectoralis by using the Hough-transform.

\subsection{Detection of the nipple}

The anatomical coordinate system is based on the skin line and the line through the nipple that is perpendicular to the pectoralis. One example of methods for nipple detection is given in [3]. This method, however, is based on intensity measures and is sensitive to digitalisation parameters [4].

In this work, we make use of the geometrical assumption that the nipple is located at the point of the boundary that is furthest away from the pectoralis. It has, however, been noted in the literature [2] that the assumption does not necessarily holds but recently we have shown that this assumption yields a rather good approximation of the nipple [15]. Based on 305 images from the MIAS database the probability of an error of zero is 0.41 and the probability of estimating the position of the nipple within the nipple is 0.62 . In no case was the position of the nipple estimated with a larger error than four times the average extension of the nipple. 


\section{Geometric accuracy}

The coordinate axes are expressed in relation to anatomical landmarks, i.e. the projected skin-air interface, a straight-line approximation of the pectoralis and the position of the nipple. It is, of course, essential to estimate how sensitive the coordinates are to disturbances in the determination of these three parameters.

In this section these errors are investigated as well as the errors associated with the movement of tissue while under compression.

\subsection{The pectoralis line}

The pectoralis line determines the direction of the nipple line since the nipple line passes through the nipple and is orthogonal to the pectoralis line. In other words, it is only interesting to determine how the angle of the pectoralis line affects the estimated coordinate. It is clear that the estimated direction of the pectoralis line does not affect the equidistance lines. From simple geometry we have, that if the nipple line is perpendicular to the equidistance lines the effect of a small disturbance of the direction is minimised and the coordinate is most sensitive when the nipple line is a tangent to the equidistance lines. This is illustrated in Figure 2 Left. In this case, a 2-degree disturbance is introduced in the two cases of normal direction and tangential direction. The distance along the dotted equidistance line between a point $p$ and the perturbed point $\hat{p}$ is clearly greater in the tangent case than in the normal case. The problem of determining the effect of a small perturbation in the direction of the pectoralis line is thus reduced to whether or not the nipple line is perpendicular to the equidistance lines or not. In [15] we used the assumption that the nipple is the point on the skin line with maximum distance to a straight line approximation of the pectoralis muscle. As pointed out earlier this yields a rather good approximation of the position of the nipple. For this to hold the nipple must, on average, be situated where a line of the same direction as the pectoralis line is a tangent to the skin-line. Since the equidistance lines are parallel to the skin-line they must, on average, be normal to the nipple line that is normal to the pectoralis line. Hence we could expect that a small perturbation would yield case $a$ in Figure 2 Left, rather than case $b$. The conclusion is that the proposed coordinates are rather stable with regards to perturbations in the estimated of the direction of the pectoralis line.

\subsection{The skin line}

The skin-line is defined to be the projected boundary between the skin and the air. The coordinates are, of course, sensitive to perturbations of this line. However, the perturbations that is likely to occur stems from the segmentation of the skin line. Moreover, it is reasonable to assume that they will not affect the shape of the skin line, only its perceived distance to the pectoralis muscle. Thus the coordinates of the same breast will be affected the same amount, but we might expect larger errors when using the coordinates for bilateral comparison. However, the error is not amplified in any way and we can expect the error to be of the same magnitude as the error in determining the skin line. 

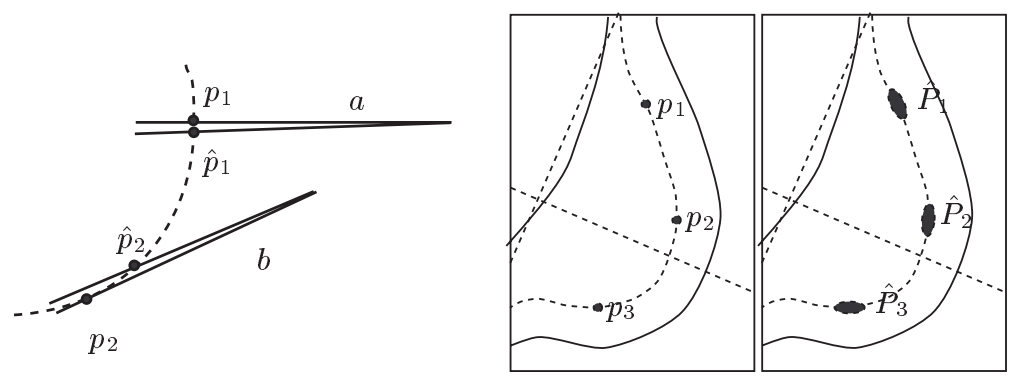

Fig. 2. Left: The effect of a 2 degree change of the direction of the nipple line in the case of $a$ ) normal direction and $b$ ) tangential direction. Right: Points $p_{i}$ and the set of their estimated locations $\hat{P}_{i}$ based on geometrical errors

\subsection{The position of the mamilla}

If the estimated position of the mamilla is wrong we could, as in the case of the skin line expect the coordinates to differ in the same magnitude as the error of the position of the mamilla. In [15] we showed that the error obtained when estimating the position of the mamilla is expected to be about 0.6 times the size of the extension of the mamilla.

\subsection{The errors in relation to each other}

The errors associated with the direction of the pectoralis muscle and the position of the mamilla are added and will affect the estimated distance along the equidistance line from the nipple line to the point of interest. The error of determining the skin line will mostly affect the distance from the point of interest to the skin line. It is reasonable to assume that we are able to determine the position of the skin line with higher accuracy than the position of the nipple. The effect of the geometric errors are illustrated in Figure 2 Right. Given a point $p_{i}$ and the problem to locate the corresponding point in a bilateral or temporal mammogram we would expect the set of points $\hat{P}_{i}$ to appear as in the figure. All points within the set $\hat{P}_{i}$ are likely to be the corresponding point to $p_{i}$. A reasonable model for the error would be to assume two dimensional Gaussian distributions with covariance aligning them to the equidistance lines.

\subsection{The effect of compression}

A course model of how tissue moves while under compression would be that it moves away from the pectoralis muscle in some direction. Two obvious candidates for directions are that the tissue moves perpendicular to the pectoralis muscle or perpendicular to the skin. The direction of these tissue movements and the effect they have on the corresponding coordinates is that the geometrical errors are smeared in the direction of the tissue movement. 

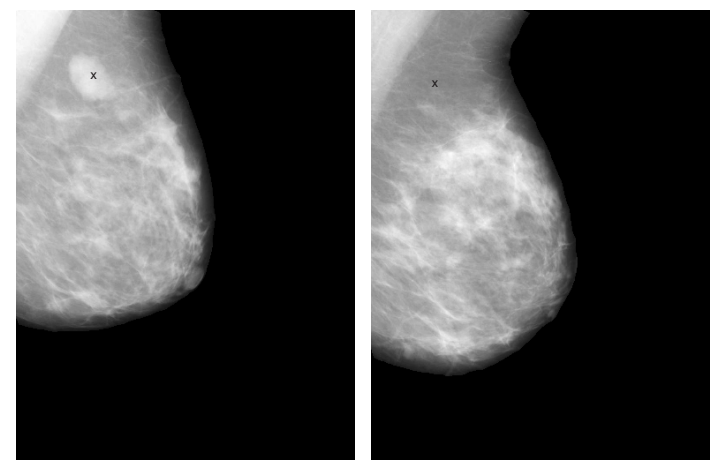

Fig. 3. Mammograms 15 and 16 from the MIAS-database. The $\times$ marked in both images has the anatomical coordinate 92 pixels to the skin line and 219 pixels above the nipple line.

\section{Results and further work}

Initial tests has been carried out on the MIAS database with encouraging results, see for instance Figures 3 and 4 . This indicates that the anatomical coordinates are useful in bilateral comparison. There is, however, large drawback with this database regarding the radiological quality of the mammograms. It is quite common not to have the entire breast visible, especially the infra mammary sulcus. Since the skin-line is obscured by the lower edge of the image, it does not work to use the equidistance lines to the skin line as a reference line.

\section{References}

1. G. Borgefors. Distance transformations in digital images. Comput. Vision, Graphics, Image Processing, 34:344-371, 1986.

2. R. Chandrasekhar. Systematic Segmentation of Mammograms. PhD thesis, The University of Western Australia, 1996.

3. R. Chandrasekhar and Y. Attikiouzel. A simple method for automatically locating the nipple on mammograms. IEEE Transactions on Medical Imaging, 16:483-494, 51997.

4. R. Chandrasekhar and Y. Attikiouzel. The need to standardize and calibrate databases of digitized mammograms. In N. Karssemeijer, M. Thijsen, J. Hendriks, and L. van Erning, editors, Digital Mammography, Computational Imaging and Vision, pages 403-404. Kluwer Academic Publishers, 1998.

5. F. Georgsson. Differential analysis of bilateral mammograms. In I. Austvoll, editor, Proceedings of The 12th Scandinavian Conference on Image Analysis, pages 70-77. 2001.

6. F. Georgsson and Niclas Björnestål. On the problem of breast compression modelling. In H.U. Lemke, M.W. Vannier, K. Inamura, A.G. Farman, K. Doi, and J.H. Reiber, editors, Computer Assisted Radiology and Surgery, pages 677-682, 2002. 

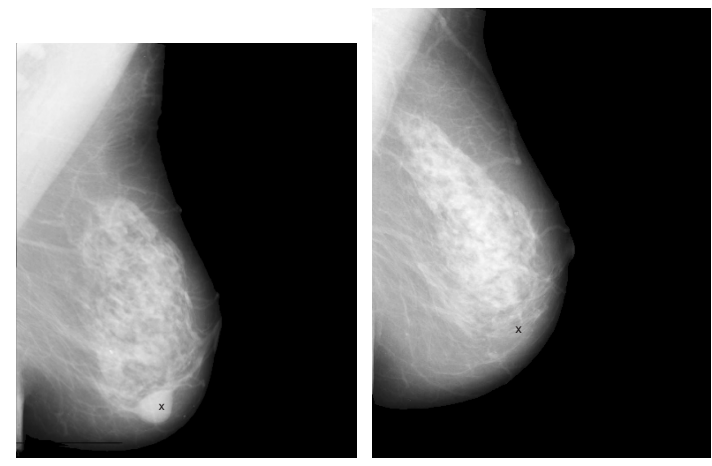

Fig. 4. Mammograms 21 and 22 from the MIAS-database. The $\times$ marked in both images has the anatomical coordinate 37 pixels below the skin line and 30 pixels to the nipple line.

7. F. Groen and P. Verbeek. Freeman code probabilities of object boundary quantized contours. Computer Graphics and Image Processing, 7:391-402, 1978.

8. N. Karssemeijer. Automated classification of parenchymal patterns in mammograms. Physics in Medicine and Biology, 43:365-378, 1998.

9. N. Karssemeijer and G. te Brake. Combining single view features and asymmetry for detection of mass lesions. In N. Karssemeijer, M. Thijsen, J. Hendriks, and L. van Erning, editors, Digital Mammography, Computational Imaging and Vision, pages 95-102. Kluwer Academic Publishers, 1998.

10. S.-L. Kok-Wiles, M. Brady, and R. Highnam. Comparing mammogram pairs for the detection of lesions. In N. Karssemeijer, M. Thijsen, J. Hendriks, and L. van Erning, editors, Digital Mammography, Computational Imaging and Vision, pages 103-110. Kluwer Academic Publishers, 1998.

11. S. Kumar and D. Goldgof. Recovery of global nonrigid motion - a model based approach without point correspondences. In Proceedings of 1996 Conference on Computer Vision and Pattern Recognition. 594-599 1996.

12. T.-K. Lau and W.F. Bischof. Automated detection of breast tumors using the asymmetry approach. Computers and Biomedical Research, 24:273-295, 1991.

13. A.J. Mendez, P.G. Tahoces, M.J. Lado, M. Souto, and J.J. Vidal. Computer-aided diagnosis: Automatic detection of malignant masses in digitized mammograms. Medical Physics, 25:957-964, 61998.

14. P. Miller and S. Astley. Automated detection of mammographic asymmetry using anatomical features. International Journal of Pattern Recognition and Artificial Intelligence, 7(6):1461-1476, 1993.

15. C. Olsén and F. Georgsson. The accuracy of geometric approximation of the mamilla in mammograms. To appear in proceedings of CARS'03, June 2003.

16. M.Y. Sallam and K.W. Bowyer. Registration and difference analysis of corresponding mammogram images. Medical Image Analysis, 3(2):103-118, 1999. 